\title{
Improving offering strategies for wind farms enhanced with storage capability
}

\author{
Ding, Huajie; Hu, Zechun; Song, Yonghua; Pinson, Pierre
}

\section{Published in:}

Proceedings of 2015 IEEE Eindhoven PowerTech

Link to article, DOI:

10.1109/PTC.2015.7232244

Publication date:

2015

Document Version

Peer reviewed version

Link back to DTU Orbit

Citation (APA):

Ding, H., Hu, Z., Song, Y., \& Pinson, P. (2015). Improving offering strategies for wind farms enhanced with storage capability. In Proceedings of 2015 IEEE Eindhoven PowerTech (pp. 1-6). IEEE.

https://doi.org/10.1109/PTC.2015.7232244

\section{General rights}

Copyright and moral rights for the publications made accessible in the public portal are retained by the authors and/or other copyright owners and it is a condition of accessing publications that users recognise and abide by the legal requirements associated with these rights.

- Users may download and print one copy of any publication from the public portal for the purpose of private study or research.

- You may not further distribute the material or use it for any profit-making activity or commercial gain

- You may freely distribute the URL identifying the publication in the public portal

If you believe that this document breaches copyright please contact us providing details, and we will remove access to the work immediately and investigate your claim 


\section{Improving Offering Strategies for Wind Farms Enhanced With Storage Capability}

\author{
Huajie Ding/ Zechun Hu/ Yonghua Song \\ Department of Electrical Engineering \\ Tsinghua University \\ Beijing, China \\ dinghj13@mails.tsinghua.edu.cn
}

\author{
Pierre Pinson \\ Department of Electrical Engineering \\ Technical University of Denmark \\ Lyngby, Denmark \\ ppin@elektro.dtu.dk
}

\begin{abstract}
Due to the flexible charging and discharging capability, energy storage system (ESS) is thought of as a promising complement to wind farms (WF) in participating into electricity markets. This paper proposes a reserve-based realtime operation strategy of ESS to make arbitrage and to alleviate the wind power deviation from day-ahead contracts. Taking into account the operation strategy as well as two-price balancing market rules, a day-ahead bidding strategy of WFESS system is put forward and formulated. A modified gradient descent algorithm is described to solve the formulations. In the case studies, the computational efficiency of the algorithm is validated firstly. Moreover, a number of scenarios with/without considering the temporal dependence of wind power forecast error are designed and employed to compare the proposed strategy with other common ones in terms of profit.
\end{abstract}

Index Terms-Bidding strategy, electricity markets, energy storage system, real-time operation, wind farm

\section{INTRODUCTION}

$\mathrm{R}$ ecent decades have witnessed the rapid development of wind power generation. It has gradually been a general consensus that the increasing capacity of wind power should be traded in day-ahead markets [1]. Based on the limited wind power forecast accuracy, optimal bidding strategies of wind farms (WF) in day-ahead electricity markets has been widely studied. Reference [1] proposes a closed-form optimal day-ahead bidding strategy, Expected Utility Maximization (EUM) Strategy. It considers stochastic prices and wind generation, and has advantages over the LP models on transparency of results, computational efficiency and data requirement. In order to extend the application of EUM strategy to situations where WFs coordinate with conventional generators, a more general loss function is put forward in [2] to express the economic loss on the WF resulting from the wind power deviation. Authors of [3] anchor the bidding amounts within a certain neighborhood of deterministic forecast values to account for risk aversion while improving the EUM strategy. It can alleviate risky bidding in electricity markets, which may result in large imbalance and is not welcome by Transmission System Operators (TSO).

The limited accuracy of wind power forecast necessarily result in the revenue loss for wind power producers as they should buy up-regulation (additional energy sold by other

The research work was supported in part by National Natural Science Foundation of China (51107060) and Chinese Scholarship Council. Pierre Pinson is partly supported by the Danish Strategic Council for Strategic Research through the projects PROAIN (no. 3045-00012B/DSF) and 5s Future Electricity Markets (no. 12-132636/DSF). participants) or sell down-regulation (energy bought by other participants) in balancing markets to meet the deviation with day-ahead contracts. The energy storage system (ESS) can be regarded as a suitable complement to the stochastic wind power, as it can inject and extract energy flexibly to accommodate the imbalance [4-6]. Furthermore, it can make arbitrage with the variable prices to increase the overall profit. The study of [7] shows that WFs and ESS can get both better off through the contract about the imbalance service. The storage studied in [8] is employed to cover the contracted shortfalls of WF. The concavity of objective functions based on the greedy operation strategy of ESS is studied, but the arbitrage strategy of storage is overlooked, which will influence the day-ahead bidding and further affect the profit. Pumped storage plant is studied to utilize the wind spillage and arbitrage between different time periods in [9]. However, the formulation does not consider the penalty of deviation, which is different from the rule of electricity market.

The first contribution of this paper is illustrating the influence of ESS on day-ahead bidding of WF. The cooperative mode is that the ESS has charging/discharging reserve capacity for each time interval, up to which the ESS can compensate for potential imbalance from WF. Based on the cooperative strategy and price-taker assumption, a mixed integer nonlinear programming model is proposed to maximize the expected profit. An additional contribution is the proposal of a computational efficient algorithm to solve the problem, which is modified from the gradient descent algorithm and validated by a case-study with three market time units. Furthermore, scenarios with different temporal dependences of wind power forecast error are generated to compare the proposed strategy with other common ones.

\section{OPTIMAL BIDDING AND OPERATION STRATEGY}

\section{A. Imbalance Management in Electricity Markets}

In most deregulated electricity markets such as the Scandinavian Nord Pool, participants should trade in both the day-ahead market and balancing market. There are also intraday markets (or adjustment markets) between day-ahead and balancing stages for transactions of renewable energy generation [10]. But as the trading amount in such markets is relatively small, it is not considered in this paper.

In the day-ahead market, power plants bid for their generation schedule with 12 to 14 hours prior to actual generation [11]. The cleared schedules are subject to deviation penalties, which means the participants need to buy or sell up/down regulation services for any deviation of actual output from schedules in balancing markets. The balancing 
markets can be divided into two categories according to whether the balancing price changes with the imbalance sign [12]. The deviation is traded at a unique price in the one-price balancing market, which is adopted in markets like Dutch APX [13]. In two-price markets such as Nord Pool and the Iberian one, deviation opposite to the system imbalance is traded at day-ahead price while the imbalance of the same sign with that of system is traded at the cleared balancing price. The model proposed in this paper is based on the second one as it is more comprehensive.

\section{B. Real-time Operation Strategy}

In most literature the ESS is used to compensate the imbalance of WF from day-ahead offers, by which the WFESS can be regarded as a conventional generation plant as a whole. However, operating ESS in this way may risk charging at high prices but discharging at low prices. Besides, the imperfect round-trip efficiency of ESS would further reduce the potential profit of the system.

In this paper, a novel operation strategy is proposed. The charging or discharging status of each time interval is optimized in day-ahead section, which means the ESS only works if the real-time imbalance sign of the WF output goes with the predetermined working status. Furthermore, it also sets constraints on the charging and discharging power, which can be thought of as the operational reserve provided by the ESS. It means that even if the deviation of WF exceeds the reserve capacity, the ESS can charge or discharge at most to the predetermined upper bounds, which are often lower than the ones determined by the operational constraints. By this method the ESS can avoid charging at high-price intervals or discharging at low-price periods.

\section{Formulations of Bidding and Operation Strategies}

As mentioned above, the objective of ESS operation is to make arbitrage and to balance the output deviation of WF. In the formulation, the wind power $p$ in interval $t$ is assumed to distribution $f_{t}(p)$. Then the expected profit of all $T$ intervals can be written as,

$$
\max \sum_{t=1}^{T} E\left[S_{t}\left(B_{t}^{D}, B S_{t}^{D}, B S_{t}^{C}\right) \mid \lambda_{t}^{D}, \lambda_{t}^{u p}, \lambda_{t}^{d w}\right] \Delta t
$$

where $\Delta t$ is the time duration of each interval. $B_{t}^{D}$ is the day-ahead bidding of WF-ESS system, and $B S_{t}^{D}, B S_{t}^{C}$ are discharging and charging capacity of ESS. As the WF-ESS system is assumed to be a price taker, the day-ahead prices $\lambda_{t}^{D}$ and up/down regulation prices $\lambda_{t}^{u p}, \lambda_{t}^{d w}$ are independent from its bidding. Consequently the stochastic prices can be presented by their expected value, and the expected profit in each interval can be expressed as,

$$
\begin{aligned}
& E\left[S_{t}\left(B_{t}^{D}, B S_{t}^{D}, B S_{t}^{C}\right) \mid \lambda_{t}^{D}, \lambda_{t}^{u p}, \lambda_{t}^{d w}\right] \\
= & \bar{\lambda}_{t}^{D} B_{t}^{D}+\bar{\lambda}_{t}^{u p} \int_{\underline{w f_{t}}}^{B_{t}^{D}-B S_{t}^{D}}\left(p-B_{t}^{D}+B S_{t}^{D}\right) f_{t}(p) d p \\
+ & \bar{\lambda}_{t}^{d w} \int_{B_{t}^{D}+B S_{t}^{C}}^{\overline{w f_{t}}}\left(p-B_{t}^{D}-B S_{t}^{C}\right) f_{t}(p) d p
\end{aligned}
$$

where $\bar{\lambda}_{t}^{D}$ is the expected day-ahead price, $\bar{\lambda}_{t}^{u p}, \bar{\lambda}_{t}^{d w}$ are expected up/down-regulation prices for interval $t$ respectively [1]. The expected profit in interval $t$ consists of three parts. The first one is the day-ahead bidding profit, the second part is the cost of purchasing up-regulation service (negative in value) and the third part is the profit of selling downregulation. Necessarily,

$$
\begin{array}{ll}
0 \leq B S_{t}^{C} \leq u_{t}^{C}\left(\overline{w f_{t}}-B_{t}^{D}\right) & \forall t \in\{1,2 \ldots T\} \\
0 \leq B S_{t}^{D} \leq u_{t}^{D}\left(B_{t}^{D}-\underline{w f_{t}}\right) & \forall t \in\{1,2 \ldots T\}
\end{array}
$$

where $\overline{w f_{t}}, \underline{w f_{t}}$ are upper and lower bounds of wind power output of interval $t$, and $u_{t}^{C}, u_{t}^{D}$ are variables indicating charging and discharging status of ESS. There are also some operational constraints of ESS, including:

$$
\begin{gathered}
0 \leq B S_{t}^{C} \leq u_{t}^{C} \overline{B S^{C}} \quad \forall t \in\{1,2 \ldots T\} \\
0 \leq B S_{t}^{D} \leq u_{t}^{D} \overline{B S^{D}} \quad \forall t \in\{1,2 \ldots T\} \\
u_{t}^{C}+u_{t}^{D} \leq 1 \quad u_{t}^{C}, u_{t}^{D} \in\{0,1\} \quad \forall t \in\{1,2 \ldots T\} \\
E_{t}=E_{0}+\sum_{j=1}^{t-1} B S_{j}^{C} \cdot \eta_{C} \cdot \Delta t-\sum_{j=1}^{t-1} B S_{j}^{D} / \eta_{D} \cdot \Delta t \quad \forall t \in\{1,2 \ldots T\} \\
E_{\min } \leq E_{t} \leq E_{\max } \quad \forall t \in\{1,2 \ldots T\} \\
E_{T}=E_{0}
\end{gathered}
$$

where (5) and (6) constrain the charging and discharging power, at interval $t$ within the operation range. Simultaneous charging and discharging is prevented by (7). The energy transition of ESS is expressed in (8) and the residual energy should be within allowable range $\left[E_{\min }, E_{\max }\right]$ at any time interval with (9). In this paper, the energy in ESS is constrained to be the same at the beginning and end of the day, as shown in (10).

\section{SOLUTION METHOD}

As integration is included in the objective function (2) and binary variables are included in (3)-(7), the formulation is a mixed integer non-linear model, and cannot be solved directly. In this paper, the gradient descent algorithm is modified according to the characteristics of the formulation. It should be noted that as the problem may be nonconvex, in that case the proposed algorithm will only get the nearest local optimal solution. However, the computational effort required for this algorithm is much lower than evolutionary algorithms, which can only theoretically get global optimal solution. The computation-friendly characteristic makes it easy to use and suitable to integrate into rolling optimization. More importantly, the algorithm can obtain the satisfactory solution by choosing the proper initial point.

\section{A. Framework of the Algorithm}

The proposed algorithm consists of following steps:

$$
\text { Initialization: }\left\{B_{t}^{D \bullet}, B S_{t}^{D \bullet}, B S_{t}^{C \bullet}\right\}=\left\{F^{-1}\left(\frac{\bar{\lambda}_{t}^{D}-\bar{\lambda}_{t}^{\alpha w}}{\bar{\lambda}_{t}^{u p}-\bar{\lambda}_{t}^{\alpha w}}\right), \mathbf{0}, \mathbf{0}\right\}
$$

Step 1: execute ESS module $\Rightarrow\left\{\left[\Delta B S_{t}^{D k}, \Delta B S_{t}^{C k}\right]\right\}$ 
Step 2: execute Bidding module $\Rightarrow\left\{\Delta B_{t}^{D k}\right\}$

Step 3: back-tracking method to determine the step:

Substep1: $\gamma=1$

Substep2:

$$
\begin{aligned}
& B S_{t}^{D k}(\gamma)=B S_{t}^{D(k-1)}+\left(1-(1-\gamma) u_{t}^{D}\right) \Delta B S_{t}^{D k} \\
& B S_{t}^{C k}(\gamma)=B S_{t}^{C(k-1)}+\left(1-(1-\gamma) u_{t}^{C}\right) \Delta B S_{t}^{C k} \\
& B_{t}^{D k}(\gamma)=B_{t}^{D(k-1)}+\gamma \Delta B_{t}^{D k} \Rightarrow S^{k}(\gamma)
\end{aligned}
$$

Substep3: while $S^{k}(\gamma)<S^{k-1}$, then $\gamma=\beta \gamma$, go to Substep2

Step 4: if $\left|S^{k}(\gamma)-S^{k-1}\right| \leq \varepsilon$, or maximal iteration steps are reached, end. Otherwise, go to Step 1

In the algorithm, the parameter $\gamma$ is the step size, whose initial value is 1 . The backtracking parameter $\beta$ is the factor which shortens the step size.

\section{B. Linear Approximation of the Objective}

In (2), when the prices are available through predictions, then the objective function is a function of $B_{t}^{D}, B S_{t}^{D}, B S_{t}^{C}$, noted as $S\left(B_{t}^{D}, B S_{t}^{D}, B S_{t}^{C}\right)$. At any interval $t$, the expected profit can be approximated as:

$$
\begin{aligned}
& S\left(B_{t}^{D}, B S_{t}^{D}, B S_{t}^{C}\right) \approx S\left(B_{t}^{D 0}, B S_{t}^{D 0}, B S_{t}^{C 0}\right) \\
& +\left[\frac{\partial S}{\partial B_{t}^{D 0}}, \frac{\partial S}{\partial B S_{t}^{D 0}}, \frac{\partial S}{\partial B S_{t}^{C 0}}\right] \cdot\left[\Delta B_{t}^{D}, \Delta B S_{t}^{D}, \Delta B S_{t}^{C}\right]^{T}
\end{aligned}
$$

and

$$
\begin{aligned}
& \frac{\partial S_{t}}{\partial B_{t}^{D}}=\bar{\lambda}_{t}^{D}-\bar{\lambda}_{t}^{u p} F\left(B_{t}^{D}-B S_{t}^{D}\right)-\bar{\lambda}_{t}^{d w}\left[1-F\left(B_{t}^{D}+B S_{t}^{C}\right)\right] \\
& \frac{\partial S_{t}}{\partial B S_{t}^{D}}=\bar{\lambda}_{t}^{u p} F\left(B_{t}^{D}-B S_{t}^{D}\right) \\
& \frac{\partial S_{t}}{\partial B S_{t}^{C}}=-\bar{\lambda}_{t}^{d w}\left[1-F\left(B_{t}^{D}+B S_{t}^{C}\right)\right]
\end{aligned},
$$

where $F(p)$ is the cumulative distribution function of wind power forecast. The first part of (11) is fixed, then the optimal solution of (11) can be obtained by solving the rest part. Besides, Equation (3) (4) are quadratic if being solved directly, but can be converted to linear ones via an iterative procedure on the decision variables involved.

\section{ESS Module}

In this module, the decision variables are all about the charging and discharging of ESS. Furthermore, the objective function only contains the ESS-related parts. The superscript $k$ means the variables are of iteration $k$, where all the parameters of iteration $(k-1)$ are constants. The formulation is,

$$
\begin{aligned}
\operatorname{Max} \sum_{t=1}^{T} & \left(\frac{\partial S_{t}}{\partial B S_{t}^{D(k-1)}} \Delta B S_{t}^{D k}+\frac{\partial S_{t}}{\partial B S_{t}^{C(k-1)}} \Delta B S_{t}^{C k}\right) \\
0 & \leq B S_{t}^{C(k-1)}+\Delta B S_{t}^{C k} \leq u_{t}^{C k} \overline{B S^{C}} \\
0 & \leq B S_{t}^{D(k-1)}+\Delta B S_{t}^{D k} \leq u_{t}^{D k} \overline{B S^{D}}
\end{aligned}
$$

$$
\begin{gathered}
0 \leq B S_{t}^{C(k-1)}+\Delta B S_{t}^{C k} \leq u_{t}^{C k}\left(\overline{w f_{t}}-B_{t}^{D(k-1)}\right) \\
0 \leq B S_{t}^{\mathrm{D}(k-1)}+\Delta B S_{t}^{D k} \leq u_{t}^{D k}\left(B_{t}^{D(k-1)}-\underline{w f_{t}}\right) \\
u_{t}^{C k}+u_{t}^{D k} \leq 1 \\
E_{t}^{k}=E_{t}^{k-1}+\sum_{j=1}^{t-1} \Delta B S_{t}^{C k} \cdot \eta_{C} \cdot \Delta t-\sum_{j=1}^{t-1} \Delta B S_{t}^{D k} / \eta_{D} \cdot \Delta t \\
E_{\min } \leq E_{t}^{k} \leq E_{\max } \\
E_{T}^{k}=E_{0}
\end{gathered}
$$

The formulation avoids the quadratic formation as $\left\{B_{t}^{D(k-1)}\right\}$ in (15) and (16) are regarded as constants.

\section{Bidding Module}

In this module, the decision variables are only biddingrelated variables, and the objective function only contains the day-ahead bidding profit. Formulations of this module are:

$$
\begin{gathered}
\operatorname{Max} \sum_{t=1}^{T} \frac{\partial S_{t}}{\partial B_{t}^{D(k-1)}} \Delta B_{t}^{D k} \\
B_{t}^{D(k-1)}+\Delta B_{t}^{D k} \leq \overline{w f_{t}}-B S_{t}^{C k-1}-\Delta B S_{t}^{C k} \\
B S_{t}^{D(k-1)}+\Delta B S_{t}^{D k}+\underline{w f_{t}} \leq B_{t}^{D(k-1)}+\Delta B_{t}^{D k} .
\end{gathered}
$$

Here the latest information of charging and discharging is considered in the bidding block to accelerate the computation.

\section{CASE STUDIES}

\section{A. Case Design}

In the case studies, only three intervals are designed for the sake of simplicity and transparency. The forecasted wind power is assumed to obey uniform distribution. As there is no limit on the distribution in (11), other distributions can also be adapted to the proposed method. The assumption of uniform is not very grounded but is the simplest, especially when integrated into objective functions. With the uniform distribution $p \rightarrow U\left(\underline{w f_{t}}, \overline{w f_{t}}\right)$ assumption embedded into (2), the objective can be expressed as,

$$
S_{t}=\bar{\lambda}_{t}^{D} B_{t}^{D}-\frac{\bar{\lambda}_{t}^{u p}}{2 \overline{w f_{t}}}\left(B_{t}^{D}-B S_{t}^{D}\right)^{2}+\frac{\bar{\lambda}_{t}^{d w}}{2 \overline{w f_{t}}}\left(\overline{w f_{t}}-B_{t}^{D}-B S_{t}^{C}\right)^{2}
$$

With constraints (3)-(10), a quadratic formulation is built up and to be solved by commercial software such as CPLEX, and can further be compared with the proposed algorithm in accuracy. Main parameters are listed in Table I and Table II.

\begin{tabular}{cccc}
\multicolumn{5}{c}{ Table I basic parameters of the case } \\
\hline$\eta_{C}$ & $\eta_{D}$ & $E_{\min }[M W h]$ & $E_{\max }[M W h]$ \\
0.9 & 0.9 & 1 & 10 \\
\hline$E_{0}[M W h]$ & $P_{\max }^{C}[M W]$ & $P_{\max }^{D}[M W]$ & $\beta$ \\
5 & 10 & 10 & 0.3 \\
\hline
\end{tabular}


Table II basic parameters of the case

\begin{tabular}{ccc}
\hline Time interval & $\bar{\lambda}^{D}[D K K / k W h]$ & $\bar{\lambda}^{d w}[D K K / k W h]$ \\
1 & 0.4 & 0.2 \\
2 & 0.8 & 0.7 \\
3 & 0.6 & 0.5 \\
\hline $\bar{\lambda}^{u p}[D K K / k W h]$ & $\overline{W f}[M W]$ & $\underline{W f}[M W]$ \\
0.5 & 90 & 0 \\
1.0 & 60 & 0 \\
0.7 & 75 & 0 \\
\hline
\end{tabular}

\section{B. Numerical Results}

From Table III one can see that with the coordination of ESS, the system tends to bid higher amount in the day-ahead market. As the price in period 2 is higher, the ESS sets some discharging reserve in this period and charging reserve in period 1 and 3 . The increasing amount of bidding in period 2 is also much higher than that of period 1 and 3 .

\begin{tabular}{l|c|c|c}
\multicolumn{4}{c}{ Table III Comparison of bidding results } \\
\hline Interval & 1 & 2 & 3 \\
\hline Bidding without ESS [MW] & 60.0 & 20.0 & 37.5 \\
\hline Bidding with ESS [MW] & 63.7 & 47.0 & 48.5 \\
\hline Charging reserve of ESS [MW] & 5.6 & 0 & 4.4 \\
\hline Discharging reserve of ESS [MW] & 0 & 8.1 & 0 \\
\hline
\end{tabular}

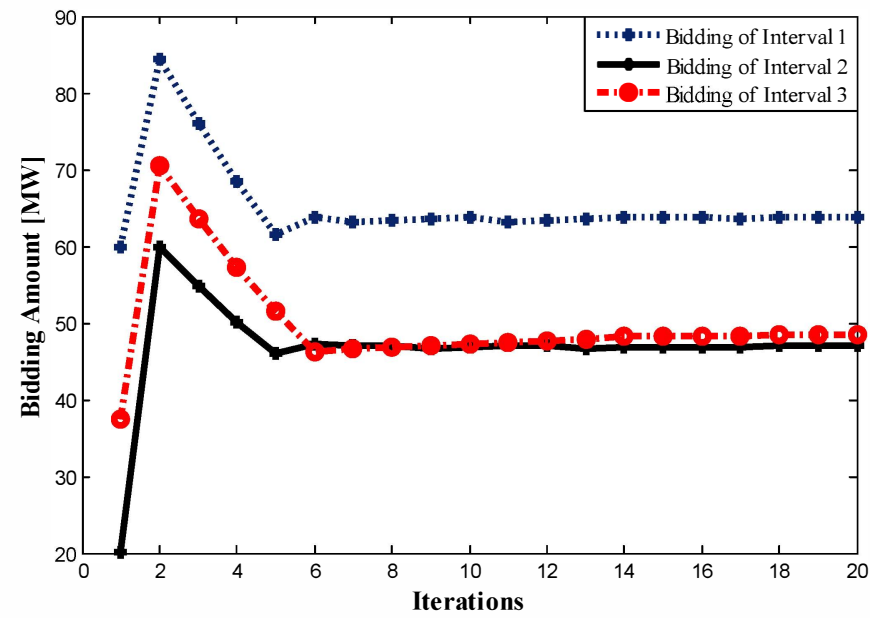

Fig. 1 Converging process of bidding in three intervals

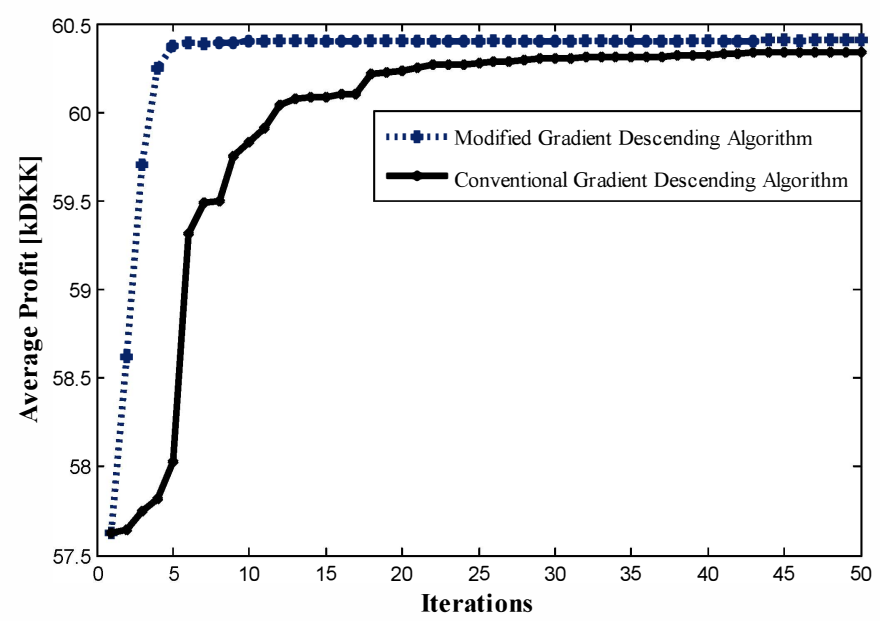

Fig. 2 Comparison on the converging process and optimality between conventional and modified gradient descent algorithms
In Fig. 1, the first iteration represents the bidding amount of all intervals without considering the storage, as done in [2]. With the iteration going up, the bidding fluctuate and gradually converge to the optimal solution. The optimal objective value is 60.41 , and has increased by $4.9 \%$ compared with that of the strategy without considering ESS. It is because that the ESS can perform arbitrage with price variance and reduce the deviation penalty of wind power, thus enhancing the expected profit.

As the latest information of charging/discharging reserve, $\Delta B S_{t}^{C k}, \Delta B S_{t}^{D k}$, is utilized in the bidding module, the converging speed of proposed algorithm is much faster than the traditional gradient descent algorithm, which only uses the information of $B S_{t}^{C(k-1)}, B S_{t}^{D(k-1)}$ in iteration $k$ (replace $\Delta B S_{t}^{C k}, \Delta B S_{t}^{D k}$ by $B S_{t}^{C(k-1)}, B S_{t}^{D(k-1)}$ in (13) and (14)). As shown in Fig. 2, it takes the modified gradient descent algorithm no more than 6 iterations to reach convergence, whose value is $60.41 \mathrm{kDKK}$. Meanwhile, it takes the conventional algorithm about 40 iterations, with the objective value of $60.34 \mathrm{kDKK}$. The numeric results show that the proposed algorithm takes advantage over traditional one both on converging speed and optimality.

The Hessian matrix of the objective function is as

$$
\begin{aligned}
H & =\left[\begin{array}{ccc}
\frac{\partial^{2} S_{t}}{\partial B_{t}^{D 2}} & \frac{\partial^{2} S_{t}}{\partial B_{t}^{D} \partial B S_{t}^{D}} & \frac{\partial^{2} S_{t}}{\partial B_{t}^{D} \partial B S_{t}^{C}} \\
\frac{\partial^{2} S_{t}}{\partial B S_{t}^{D} \partial B_{t}^{D}} & \frac{\partial^{2} S_{t}}{\partial B S_{t}^{D 2}} & \frac{\partial^{2} S_{t}}{\partial B S_{t}^{D} \partial B S_{t}^{C}} \\
\frac{\partial^{2} S_{t}}{\partial B S_{t}^{C} \partial B_{t}^{D}} & \frac{\partial^{2} S_{t}}{\partial B S_{t}^{C} \partial B S_{t}^{D}} & \frac{\partial^{2} S_{t}}{\partial B S_{t}^{C 2}}
\end{array}\right] \\
& =\left[\begin{array}{ccc}
-\frac{\lambda_{t}^{u p}-\lambda_{t}^{d w}}{\overline{w f}} & \frac{\lambda_{t}^{u p}}{\overline{w f}} & \frac{\lambda_{t}^{d w}}{\overline{w f}} \\
\frac{-\lambda_{t}^{u p}}{\overline{w f}} & \frac{-\lambda_{t}^{u p}}{\overline{w f}} & 0 \\
\frac{\lambda_{t}^{d w}}{\overline{w f}} & 0 & \frac{\lambda_{t}^{d w}}{\overline{w f}}
\end{array}\right]
\end{aligned}
$$

As the third order leading principal minor of (25) is positive, the Hessian matrix of the objective function (2) is not negative definite. It means the problem is nonconvex, and CPLEX only gets the upper bound, which is $60.60 \mathrm{kDKK}$. Meanwhile, the answer got from the proposed algorithm is $60.41 \mathrm{kDKK}$, which is quite near the upper bound.

\section{Influence of Wind Power Correlation}

In the optimization model, the wind power generation of each interval is assumed to obey specific distribution independently. However, the wind power generation at interval $t$ will partially depend on that of interval $(t-1)$. In order to consider the influence of correlation of wind power generation, two sets of scenarios are generated to compare. In the first set of scenarios, wind power of each interval is generated independently and only obeys the distribution for the interval. In the second set of scenarios, the method 
proposed in [14], [15] is applied to generate scenarios, where the correlation and distribution are considered simultaneously. In the scenario-generation method, information of predictive distributions of each time interval, $\widehat{f}(t+k \mid t)$, and covariance matrix $\Sigma$ are necessary. The predictive distribution is given as uniform distribution and the covariance matrix should be estimated with updated data. In this paper we only intend to reveal the influence of correlation on profit, so a simplified covariance matrix is used in the scenarios generation as following,

$$
\sigma_{i, j}=\alpha^{|i-j|}
$$

where $\alpha$ indicates the correlation of wind power between neighboring intervals and $\alpha \in(0,1)$.

Consequently, there are two tests according to whether temporal dependence of wind power generation between intervals is considered in scenarios. For either test, ten cases are designed and 10000 scenarios are generated for each case. Meanwhile, four bidding and operation strategies listed below are simulated and compared.

> Strategy 1: the WF works alone, and the optimal bidding of interval $t$ is $\left\{F^{-1}\left(\frac{\bar{\lambda}_{t}^{D}-\bar{\lambda}_{t}^{d w}}{\bar{\lambda}_{t}^{u p}-\bar{\lambda}_{t}^{d w}}\right)\right\}$. The WF does not adjust its output and gets settlement in balancing markets [2].

$>$ Strategy 2: WF-ESS system bids as Strategy 1, but uses the ESS to compensate the imbalance from WF as much as possible [6].

$>$ Strategy 3: WF-ESS system bids as Strategy 1, but sets operation reserve (as Table III) for the charging and discharging of ESS [8].

$>$ Strategy 4: WF-ESS system adopts the bidding strategy proposed in this paper, and sets upper bound of charging and discharging for ESS power, as Strategy 3 .

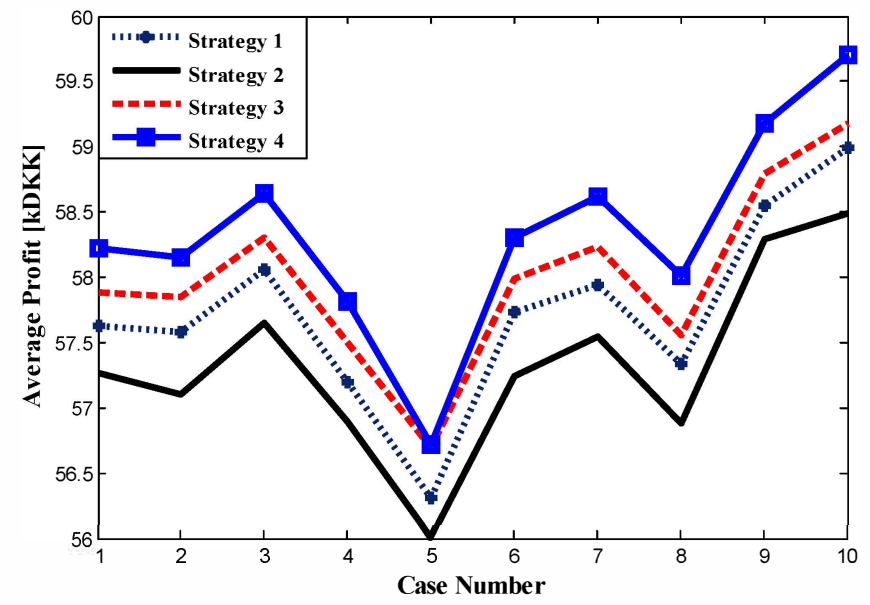

Fig. 3 Test results of uncorrelated scenarios

The results obtained from the test without considering wind power correlation between neighboring time intervals are demonstrated in Fig. 3, in form of average profit of 10000 scenarios for each strategy and case. From Fig. 3 one can observe that Strategy 4 gets much higher profit than other strategies, and Strategy 2 always has the least profit, even less than that of Strategy 1, where the WF works alone. The results come from the fact that Strategy 2 ignores the price variance, thus transiting wind energy from high-price intervals to low-price intervals in some scenarios. Besides, the round-trip efficiency of ESS will also influence the arbitrage effect.

Fig. 4 demonstrates the test results of cases considering the dependency of wind power generation. As the model and strategy proposed in this paper is built without correlation considered, Strategy 4 gets similar profit with Strategy 3. It means the improvements in profit come mostly from the realtime operation strategy of ESS. The average profit of Strategy 4 in test 2 is $56.48 \mathrm{kDKK}$, a little less than that in test 1 , where the value is $58.01 \mathrm{kDKK}$. This comparison on one hand means the correlation of wind power forecast error has influence on the performance of ESS [16], on the other hand implies the ignorance of correlation in strategy brought about losses less than 3\% $(\alpha=0.6)$.

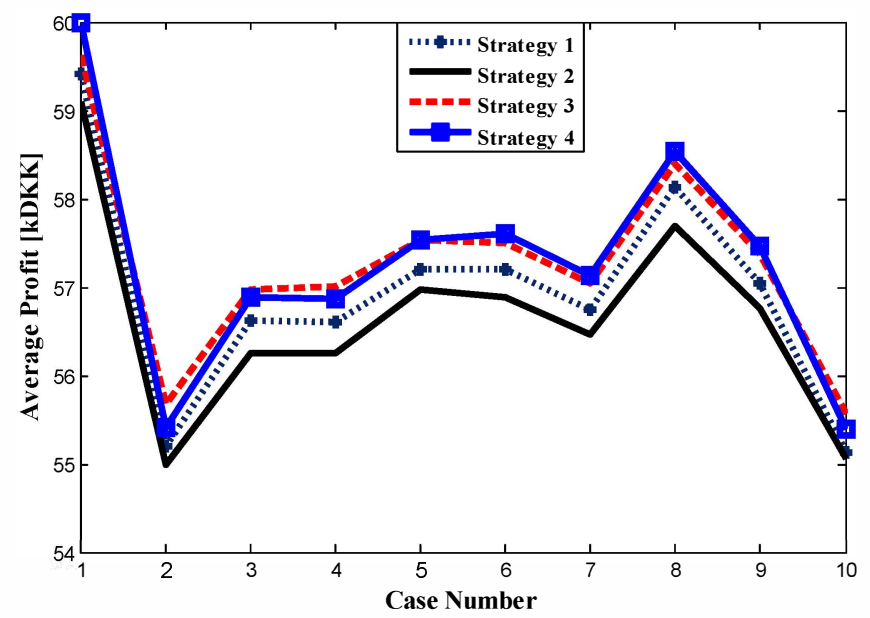

Fig. 4 Test results of correlated scenarios ( $\alpha=0.6)$

Once again the balancing strategy of ESS (Strategy 2) works worse even than independent-working strategy of WF (Strategy 1). However, it should be emphasized that the conclusion obtained here relies heavily on the price profile, and does not mean Strategy 2 is ineffective for practical operation. In fact, due to the simple and clear logic the filter strategy has been widely adapted in practice.

\section{CONCLUSION AND DISCUSSION}

This paper puts forward a novel bidding and operation strategy for the WF enhanced by the ESS. Meanwhile, a modified gradient descent algorithm is proposed to obtain the optimal solutions. Compared with the conventional gradient descent algorithm, the algorithm put forward in this paper converges faster and gets better optimality. Case studies show that the strategy can increase the total profit prominently. Besides, the proposed bidding and operation strategy is proved to have much advantage over other balancing strategies in increasing the overall profit. 
As the model is built without considering the correlation of wind power between time intervals, the profit improvement mostly results from the reserve-based operation strategy. In future work the operation strategy of ESS and WF will be further improved and the correlation of wind power will be considered in the bidding strategy.

\section{REFERENCES}

[1] C. J. Dent, J. W. Bialek and B. F. Hobbs, "Opportunity cost bidding by wind generators in forward markets: analytical results," IEEE Trans. Power Systems, vol. 26, pp. 1600-1608, 2011.

[2] P. Pinson, C. Chevallier and G. N. Kariniotakis, "Trading wind generation from short-term probabilistic forecasts of wind power," IEEE Trans. Power Systems, vol. 22, pp. 1148-1156, 2007.

[3] M. Zugno, T. J O Nsson and P. Pinson, "Trading wind energy on the basis of probabilistic forecasts both of wind generation and of market quantities," Wind Energy, vol. 16, pp. 909-926, 2013.

[4] P. Pinson, G. Papaefthymiou, B. Klockl, and J. Verboomen, "Dynamic sizing of energy storage for hedging wind power forecast uncertainty,", in Proc 2009 IEEE PES General Meeting, pp. 1-8.

[5] H. Ding, Z. Hu and Y. Song, "Rolling optimization of wind farm and energy storage system in electricity markets," IEEE Trans. Power Systems, available online.

[6] E. D. Castronuovo, J. Usaola, R. Bessa, M. Matos, I. C. Costa, L. Bremermann, J. Lugaro, and G. Kariniotakis, "An integrated approach for optimal coordination of wind power and hydro pumping storage," Wind Energy, vol. 17, pp. 829-852, 2014.

[7] G. N. Bathurst and G. Strbac, "Value of combining energy storage and wind in short-term energy and balancing markets," Electric Power Systems Research, vol. 67, pp. 1-8, 2003.

[8] E. Bitar, R. Rajagopal, P. Khargonekar, and K. Poolla, "The role of co- located storage for wind power producers in conventional electricity markets," in Proc 2011 American Operation Conference (ACC), pp. 3886-3891.

[9] E. D. Castronuovo and J. A. Peas Lopes, "On the optimization of the daily operation of a wind-hydro power plant," IEEE Trans. Power Systems, vol. 19, pp. 1599-1606, 2004

[10] H. Ding, Z. Hu and Y. Song, "Optimal intra-day coordination of wind farm and pumped-hydro-storage plant," 2014 PES General Meeting, pp. $1-5$.

[11] J. Garcia-Gonzalez, R. M. R. de la Muela, L. M. Santos, and A. M. Gonzalez, "Stochastic joint optimization of wind generation and pumped-storage units in an electricity market," IEEE Trans. Power Systems, vol. 23, pp. 460-468, 2008

[12] J. M. Morales González, A. J. Conejo, H. Madsen, P. Pinson, and M. Zugno, Integrating renewables in electricity markets: operational problems: Springer, 2014.

[13] M. Zugno, J. M. Morales, P. Pinson, and H. Madsen, "Pool strategy of a price-maker wind power producer," IEEE Trans. Power Systems, vol. 28, pp. 3440-3450, 2013.

[14] P. Pinson, H. Madsen, H. A. Nielsen, G. Papaefthymiou, and B. Klöckl, "From probabilistic forecasts to statistical scenarios of short-term wind power production," Wind energy, vol. 12, pp. 51-62, 2009

[15] A. L. J. Duque, E. D. Castronuovo, I. S A Nchez, and J. Usaola, "Optimal operation of a pumped-storage hydro plant that compensates the imbalances of a wind power producer," Electric power systems research, vol. 81, pp. 1767-1777, 2011.

[16] P. Haessig, B. Multon, H. Ben Ahmed, S. E. P. Lascaud, and P. Bondon, "Energy storage sizing for wind power: impact of the autocorrelation of day-ahead forecast errors," Wind Energy, 2013. 